\title{
Dynamics of Brane-World Cosmological Models
}

\author{
A. A. Coley ${ }^{1, *}$ \\ ${ }^{1}$ Department of Mathematics and Statistics, Dalhousie University, Halifax, Nova Scotia
}

\begin{abstract}
We show that generically the initial singularity is isotropic in spatially homogeneous cosmological models in the brane-world scenario. We then argue that it is plausible that the initial singularity is isotropic in typical brane world cosmological models. Therefore, brane cosmology naturally gives rise to a set of initial data that provide the conditions for inflation to subsequently take place, thereby solving the initial conditions problem and leading to a self-consistent and viable cosmology.
\end{abstract}

98.80.Cq

\section{INTRODUCTION}

Recent developments in string theory suggest that gravity may be a truly higher-dimensional theory, becoming effectively 4-dimensional at lower energies. This leads to modifications to Einstein's theory of general relativity (GR) at high energies and particularly at early times. There is currently great interest in higher-dimensional gravity theories inspired by string theory in which the matter fields are confined to a 3-dimensional 'braneworld' embedded in $1+3+d$ dimensions, while the gravitational field can also propagate in the $d$ extra dimensions (i.e., in the 'bulk') [1]. In this paradigm it is not necessary for the $d$ extra dimensions to be small or even compact, a departure from the standard Kaluza-Klein scenario. In recent work Randall and Sundrum [2] have shown that for $d=1$, gravity can be localized on a single 3-brane even when the fifth dimension is infinite. An elegant geometric formulation and generalization of the class of Randall-Sundrum-type brane-world models has been given in [3].

The dynamical equations on the 3-brane differ from the GR equations by terms that carry the effects of imbedding and of the free gravitational field in the 5dimensional bulk. The local (quadratic) matter fields corrections are significant only at very high energies. In addition, there are nonlocal effects from the free gravitational field in the bulk, transmitted via the projection $\mathcal{E}_{\mu \nu}$ of the bulk Weyl tensor, that contribute further corrections to the Einstein equations on the brane. $\mathcal{E}_{\mu \nu}$ can be irreducibly decomposed (with respect to a timelike congruence $u^{\mu}$ ) in terms of an effective nonlocal energy density on the brane, $\mathcal{U}$, arising from the free gravitational field in the bulk, an effective nonlocal anisotropic stress, $\mathcal{P}_{\mu \nu}$, on the brane, and an effective nonlocal energy flux on the brane, $\mathcal{Q}_{\mu}$ 断.

In general, the conservation equations do not determine all of the independent components of $\mathcal{E}_{\mu \nu}$ on the brane; in particular, there is no evolution equation for $\mathcal{P}_{\mu \nu}$. Thus in general, the projection of the 5-dimensional field equations onto the brane does not lead to a closed system. If the induced metric on the brane is flat, and the bulk is anti-de Sitter, as in the original Randall-Sundrum scenario [2], then $\mathcal{E}_{\mu \nu}=0$. More importantly, in cosmology the background induced metric is not flat, but a spatially homogeneous and isotropic Robertson-Walker (RW) 'Friedmann' model, for which

$$
\mathrm{D}_{\mu} \mathcal{U}=\mathcal{Q}_{\mu}=\mathcal{P}_{\mu \nu}=0
$$

where $\mathrm{D}_{\mu}$ is the totally projected part of the brane covariant derivative. Hence, in such cosmological settings the evolution of $\mathcal{E}_{\mu \nu}$ is fully determined, and the system of equations is closed. In general $\mathcal{U} \neq 0$ in the Friedmann background. Much effort is currently being devoted to understand the cosmology of the brane world scenario, and the Friedmann brane models have been extensively investigated (5,66).

Cosmological observations indicate that we live in a Universe which is remarkably uniform on very large scales. However, the spatial homogeneity and isotropy of the Universe is difficult to explain within the standard GR framework since, in the presence of matter, the class of solutions to the Einstein equations which evolve towards a RW universe is essentially a set of measure zero [7]. In the inflationary scenario, we live in an isotropic region of a potentially highly irregular universe as the result of an expansion phase in the early universe thereby solving many of the problems of cosmology. Thus this scenario can successfully generate a homogeneous and isotropic RW-like universe from initial conditions which, in the absence of inflation, would have resulted in a universe far removed from the one we live in today. However, still only a restricted set of initial data will lead to smooth enough conditions for the onset of inflation (i.e., the so-called cosmic no-hair theorems only apply to non-generic models [8]), so the issue of homogenization and isotropization is still not satisfactorily solved. Indeed, the initial conditions problem, that is to explain why the Universe so isotropic and spatial homogeneous from generic initial conditions, is one of the central problems of modern theoretical cosmology. We would like to revisit these issues in the context of brane cosmology. 


\section{A. Governing equations}

The field equations induced on the brane, using the Gauss-Codazzi equations, matching conditions and $Z_{2}$ symmetry, were derived in [3,4], resulting in a modification of the standard GR equations with the new terms carrying bulk effects onto the brane:

$$
G_{\mu \nu}=-\Lambda g_{\mu \nu}+\kappa^{2} T_{\mu \nu}+\widetilde{\kappa}^{4} S_{\mu \nu}-\mathcal{E}_{\mu \nu} \equiv \kappa^{2} T_{\mu \nu}^{\mathrm{tot}},
$$

where $\kappa^{2}=8 \pi / M_{\mathrm{p}}^{2}, \lambda \equiv 6 \kappa^{2} / \widetilde{\kappa}^{4}$. The generalized Friedmann equation, which determines the expansion of the universe, in the case of spatially homogeneous cosmological models is given by

$$
H^{2}=\frac{1}{3} \kappa^{2} \rho\left(1+\frac{\rho}{2 \lambda}\right)-\frac{1}{6}{ }^{3} R+\frac{1}{3} \sigma^{2}+\frac{1}{3} \Lambda+\frac{2 \mathcal{U}}{\lambda \kappa^{2}},
$$

where ${ }^{3} R$ is the scalar curvature of the hypersurfaces orthogonal to the fluid flow, which we associate with the cosmological fluid, $2 \sigma^{2} \equiv \sigma^{a b} \sigma_{a b}$ is the shear scalar, and $H=\dot{a} / a$ is the mean Hubble expansion parameter.

The brane energy-momentum tensor for a perfect fluid or a minimally-coupled scalar field is given by

$$
T_{\mu \nu}=\rho u_{\mu} u_{\nu}+p h_{\mu \nu} .
$$

where $u^{\mu}$ is the 4-velocity, $\rho$ and $p$ are the energy density and isotropic pressure, and $h_{\mu \nu} \equiv g_{\mu \nu}+u_{\mu} u_{\nu}$. It follows [3] that the brane energy-momentum tensor separately satisfies the conservation equation,

$$
\dot{\rho}+3 H(\rho+p)=0 \text {. }
$$

For a minimally coupled scalar field the energy density and pressure are, respectively,

$$
\rho=\frac{1}{2} \dot{\phi}^{2}+V(\phi), p=\frac{1}{2} \dot{\phi}^{2}-V(\phi),
$$

and Eqn (5) is equivalent to the Klein-Gordon equation.

There are many reasons to consider cosmological models that are more general than RW, both spatially homogeneous and anisotropic, and spatially inhomogeneous.

(i) The 3-curvature in RW models is given by ${ }^{3} R=$ $6 k / a^{2}$, where $k=0, \pm 1$ is the curvature constant. An equivalent 3 -curvature occurs in spatially homogeneous and isotropic curvature models, and a similar term occurs in other cosmological models.

(ii) For BRW models, equation (11) implies that $\mathcal{U}=$ $\mathcal{U}(t)$. In the models of Bianchi type I, $\mathcal{Q}_{a}=0$ but there is no restriction on $\mathcal{P}_{a b}$; however, the particular case in which this term is zero can be studied. Thus, in RW and Bianchi I models the evolution equation for $\mathcal{U}$ is 4

$$
\dot{\mathcal{U}}+4 H \mathcal{U}=0
$$

which integrates to $\mathcal{U}=\mathcal{U}_{0} / a^{4}$, which has the structure of a 'dark' radiation fluid, where $\mathcal{U}_{0}$ can be negative. (iii) A Bianchi I brane is covariantly characterized in [9]. The conservation equations reduce to Eqn (5), an evolution equation for $\mathcal{U}$ and a differential constraint on $\mathcal{P}_{\mu \nu}$. The presence of $\mathcal{P}_{\mu \nu}$ in the governing equations means that in general we cannot integrate to find the shear as in GR. However, when the nonlocal energy density vanishes or is negligible, i.e., $\mathcal{U}=0$, then the conservation equations imply $\sigma^{\mu \nu} \mathcal{P}_{\mu \nu}=0$, which is consistent on the brane [4]. This assumption is often made in the case of RW branes [6]. The shear evolution equation may then be integrated to give

$$
\sigma^{\mu \nu} \sigma_{\mu \nu}=\frac{6 \Sigma^{2}}{a^{6}}, \quad \dot{\Sigma}=0
$$

Bianchi I models on the brane have been studied by a number of authors [9,15]. A similar shear term occurs in other hypersurface orthogonal Bianchi cosmological models (such as Bianchi type $\mathrm{V}$ models).

(iv) We assume that the matter content is a non-tilting perfect fluid with a linear barotropic equation of state, i.e., $p=(\gamma-1) \rho$, where the energy conditions imply $\rho \geq 0$, and the constant $\gamma$ satisfies $\gamma \in[0,2]$. A dynamical analysis of scalar field models indicates that at early times the scalar field is effectively massless. A massless scalar field is equivalent to a perfect fluid with a stiff equation of state parameter $\gamma=2$. Eqn (5) then yields $\rho=\rho_{0} a^{-3 \gamma}$, where $\rho_{0}>0$.

We can therefore write down a phenomenological generalized Friedmann equation:

$$
H^{2}=\frac{\kappa^{2} \rho_{0}}{3 a^{3 \gamma}}+\frac{\kappa^{2} \rho_{0}^{2}}{6 \lambda a^{6 \gamma}}-\frac{k}{a^{2}}+\frac{1}{3} \Lambda+\frac{\Sigma^{2}}{a^{6}}+\frac{\mathcal{U}_{0}}{a^{4}} .
$$

This equation is applicable in a wide class of spatially homogeneous cosmological models; in particular, it is valid in Bianchi I brane models. In many applications the four-dimensional cosmological constant is assumed to be zero [2]; here we shall assume that if it is non-zero it is positive, i.e. $\Lambda \geq 0$.

\section{EARLY UNIVERSE:}

It is of considerable interest to study the classical dynamical effects in these cosmological models. A unique feature of brane cosmology is that $\rho^{2}$ dominates at early times which will lead to completely different behaviour to that in GR. First, there is the question of the existence of singularities. The generalized Raychaudhuri equation governs gravitational collapse and initial singularity behaviour on the brane. The local energy density and pressure corrections further enhance the tendency to collapse (if $2 \rho+3 p>0$ ) 10. The nonlocal term can act either way depending on its sign; a negative $\mathcal{U}$ enhances the localization of the gravitational field on the brane (the singularity can be avoided in this case), and a positive $\mathcal{U}$ acts against localization, and also reinforces the tendency to collapse. For the models governed by 
Eqn (9), it is easy to show that once a critical value of $a$ is attained, a singularity must occur.

As in GR the powerful singularity theorems of Penrose and Hawking, which guarantee the existence of spacetime singularities, will be generally applicable in brane world models. However, these theorems give little information about the nature of the singularities they predict. The most detailed proposal for the structure of spacetime singularities in GR are the conjectures of Belinskii, Khalatnikov and Lifshitz (BKL) 11] which essentially consist of two parts. The first is: I. Perfect fluid spacetimes (with a linear equation of state) with non-stiff matter have the property that asymptotically close to the singularity, matter is not dynamically significant. In the case of spatially homogeneous models this implies that spacetimes are space-like and oscillatory (asymptotic Mixmaster behaviour, oscillating indefinitely as the cosmological initial singularity is approached into the past), or are spacelike and non-oscillatory (and asymptotically Kasner at the singularity). In the case of stiff matter, which includes the massless scalar field case, the matter is not insignificant near the singularity and generically the spacetimes have singularities which are space-like and nonoscillatory (asymptotically of Jacobs form).

Theoretical justification for part I of the BKL conjectures in Bianchi models, in which the Einstein equations are a system of ODE's, has been provided using dynamical systems methods 12 and more rigorous methods [13. There are, however, special classes of models that do not obey the BKL conjectures in GR, the most important being models with an isotropic initial singularity [14, whose evolution near the cosmological initial singularity is approximated (in an appropriately defined mathematical sense) by the flat Friedmann model.

An essential feature of the brane models governed by Eqn (9) is that at high densities the term $\left(\kappa^{2} \rho_{0}^{2} / 6 \lambda a^{6 \gamma}\right)$ dominates and the the effective equation of state becomes ultra stiff. Consequently matter dominates the shear and curvature (and the other) terms in Eqn (9) when $\gamma>1$, leading to isotropic expansion of the early universe in such cases. In particular, $\sigma^{2} / H^{2} \rightarrow 0$ as $t \rightarrow 0$.

Indeed, the flat, spatially homogeneous and isotropic non-GR brane-world (without brane tension) BRW model, denoted here by $\mathcal{F}_{b}$, in which $a(t) \sim t^{\frac{1}{3 \gamma}}$ []], is always a source/repeller for $\gamma \geq 1$ (as can clearly be seen from Eqn (9)). These BRW models are valid as the initial singularity is approached $(t \rightarrow 0)$, and therefore for all physically relevant values of $\gamma$ the singularity is isotropic [14]. We expect this to be a generic feature of more general cosmological models in the brane-world scenario, as we will discuss this further below.

From Eqn (9), in the brane-world scenario anisotropy dominates at early times only for $\gamma<1$ (whereas in GR it dominates for $\gamma<2$ !), in which case the repellers are the usual anisotropic Kasner models. In the absence of shear, the BRW models are sources for $\gamma \geq 1 / 3$ when $\mathcal{U}=0$ and $\gamma \geq 2 / 3$ when $\mathcal{U} \neq 0$ [15]. The fact that the initial singularity is isotropic in Bianchi type I and V models was noted in [9, 15 .

The particular models we have considered, in which the curvature and shear are given by the expressions in the above phenomenological Eqn (9), are special. In particular, the Bianchi I and V models are not generic, and so the study of the dynamics of these models does not shed light on the typical behaviour of spatially homogeneous brane models. In order to do this we shall next consider the general Bianchi type IX (paradigm) model.

The matter corrections to the Einstein equations on the brane are given by

$$
S_{\mu \nu}=\frac{1}{12} \rho^{2} u_{\mu} u_{\nu}+\frac{1}{12} \rho(\rho+2 p) h_{\mu \nu},
$$

for a perfect fluid (or minimally-coupled scalar field). There are also nonlocal effects from the free gravitational field in the bulk via $\mathcal{U}$. All of the bulk corrections may be consolidated into an effective total energy density via Eqn (2), so that the modified Einstein equations take on the standard perfect fluid form with

$\rho^{\text {tot }}=\rho+\frac{\rho^{2}}{2 \lambda}+\frac{\widetilde{\kappa}^{4} \mathcal{U}}{\kappa^{6}}+\frac{\Lambda}{\kappa^{2}}, p^{\text {tot }}=p+\frac{\rho^{2}}{2 \lambda}+\frac{\rho p}{\lambda}-\frac{\widetilde{\kappa}^{4} \mathcal{U}}{3 \kappa^{6}}-\frac{\Lambda}{\kappa^{2}}$.

For the Bianchi IX models with $\gamma_{i j}=\operatorname{diag}\left(a_{1}^{2}, a_{2}^{2}, a_{3}^{2}\right)$, where the mean scale factor is given in terms of the three spatial scales by $a^{3}=a_{1} a_{2} a_{3}$, it can be proven [17 from the conservation Eqn (5), the nonlocal conservation Eqn (7), and the perfect fluid generalized Raychaudhuri equation that there is a singularity (at $t=0$ ), and that $\rho \rightarrow \infty$ as $t \rightarrow 0$. Close to singularity we have that $\dot{H}<0$, and

$$
\rho^{\text {tot }}=\rho^{\text {eff }}=\frac{1}{2 \lambda} \rho^{2}, \quad p^{\text {tot }}=p^{\text {eff }}=\frac{(2 \gamma-1)}{2 \lambda} \rho^{2} .
$$

The Bianchi IX equations of motion then become

$$
\left(\ln a_{1}^{2}\right)^{\prime \prime}-\left(a_{2}^{2}-a_{3}^{2}\right)^{2}+a_{1}^{4}=\frac{\kappa^{2}(1-\gamma)}{\lambda} a^{6} \rho^{2}, \text { et cyc. }
$$

where a prime denotes differentiation with respect to $\tau$ (where $d t=a^{3} d \tau$ so that $\tau \rightarrow-\infty$ as the singularity is approached), and et cyc. denotes two more equations obtained by cycling the indices of $a_{i}$ on the left-hand-side of Eqn (11) (the right-hand-side remains the same). As in the GR case, there exists a first integral

$$
\left(\ln a_{1}^{2}\right)^{\prime}\left(\ln a_{2}^{2}\right)^{\prime}+2 a_{1}^{2} a_{2}^{2}-a_{1}^{4}+c y c .=\frac{\kappa^{2}}{8 \lambda} a^{6} \rho^{2} .
$$

The BRW solution $\mathcal{F}_{b}, a_{1}=a_{2}=a_{3}=a \equiv a_{b}$ (with $\left.a_{b}(t)=t^{\frac{1}{3 \gamma}}\right)$, occurs as $\tau \rightarrow-\infty(t \rightarrow 0)$ and $a \rightarrow \infty$. From the governing equations (where we use Eqn (12) to eliminate $\kappa^{2} \rho^{2} / \lambda$ in Eqns (11) and the Raychaudhuri equation governs the evolution of $H$ ), we obtain the following 5 essential eigenvalues for the linearization about $\mathcal{F}_{b}$ :

$$
3(\gamma-1), 3(\gamma-1),(3 \gamma-1),(3 \gamma-1),(3 \gamma-1)
$$


(the first two eigenvalues correspond to shear modes while the last three correspond to curvature modes). Thus $\mathcal{F}_{b}$ is a past-attractor (repeller/source) of the Bianchi type IX models for $\gamma>1$ (for $\gamma=1$ the BRW solution is an analogue of the Jacobs stiff fluid solution in GR and is a past-attractor). There are no other pastattractors [17]; in particular, the analogues of the non-flat Kasner vacuum solutions are saddles. Hence the singularity is non-oscillatory and isotropic. It can be shown that $\mathcal{F}_{b}$ is a source in all Bianchi models [17].

The results are incomplete in that a description of the gravitational field in the bulk is not provided. Unfortunately, the evolution of the anisotropic stress part is not determined on the brane. These nonlocal terms also enter into crucial dynamical equations, such as the Raychaudhuri equation and the shear propagation equation, and can lead to important effects. The correction terms must be consistently derived from the higher-dimensional equations. Additional modifications occur for more general higher-dimensional (bulk) geometry, higher curvature corrections, higher-dimensional matter fields in the bulk, and for motion of the brane.

\section{DISCUSSION}

Consequently, we have shown that generically the initial singularity is isotropic in spatially homogeneous brane world cosmological models.

The second part of the BKL conjectures is: II. Each spatial point evolves towards the singularity as if it were a spatially homogeneous cosmology. That is, generic spacetimes have the property that spatial points decouple near the singularity and the Einstein equations effectively reduce to ODEs, so that the local dynamical behaviour is asymptotically like Bianchi models near the singularity. Therefore, according to the BKL conjectures, the singularities in general four-dimensional spacetimes in GR are space--like and oscillatory or are space-like and nonoscillatory (e.g., for massless scalar fields).

An analysis of the behaviour of spatially inhomogeneous solutions to Einstein's equations near an initial singularity is in its infancy, and hence there is less support for part II of the BKL conjectures. However, a special class of Abelian $G_{2}$ spatially inhomogeneous models were analysed and it was found that the evolution at different spatial points approach that of different Kasner solutions [18]. A recent numerical investigation of a class of vacuum Gowdy $G_{2}$ cosmological spacetimes has shown evidence that at a generic point in space the evolution towards the initial singularity is asymptotically that of a spatially homogeneous spacetime with Mixmaster behavior 19]. In both of these cases the presence of the inhomogeneity ceases to govern the dynamics asymptotically toward the singularity, thereby providing further support for the BKL conjectures.

In addition, in a recent qualitative analysis of a class of spatially inhomogeneous $G_{2}$ brane cosmological models (with one spatial degree of freedom) near the initial cosmological singularity, it was found that $\mathcal{F}_{b}$ is again a local source 17]. It was also argued, based upon local dynamical considerations and physical arguments, that the main result that the singularity is isotropic will persist when additional, more general, affects are included.

Thus it is plausible, from the BKL conjectures in GR and from this recent study of spatially inhomogeneous brane models, that typically the initial singularity is isotropic in brane world cosmological models.

Therefore, unlike the situation in GR, it is plausible that a wide range of brane cosmological models (and of non-zero measure) admit an isotropic singularity. Such a 'quiescent' cosmology 20, in which the universe began in a highly regular state but subsequently evolved towards irregularity, might offer an explanation of why our Universe might have began its evolution in such a smooth manner and may provide a realisation of Penrose's ideas on gravitational entropy and the second law of thermodynamics in cosmology 21 .

More importantly, it is therefore possible that a quiescent cosmological period occuring in brane cosmology provides a physical scenario in which the universe starts off smooth and that naturally gives rise to the conditions for inflation to subsequently take place. As noted earlier, in the conventional scenario only a restricted set of initial data will lead to the conditions possible for inflation to occur. We thus argue that brane cosmology in tandem with inflation may lead to a fully self-consistent and physically viable cosmology 22].

Subsequently, the models evolve essentially as in standard cosmology. The modified Friedmann equation for a flat RW brane (with $\Lambda=0=\mathcal{U}$ ) and the Klein-Gordon Eqn (5) yield $\dot{\rho}=-3 \dot{\phi}^{2} \rho$ at early times for a scalar field source, so that $\rho$ is monotonically decreasing and the models will eventually evolve to the low density regime.

The intermediate dynamics is affected by the brane corrections. The issue of inflation on the brane was investigated in [10], where it was shown that on an RW brane in 5-dimensional anti de Sitter space the quadratic term in $\rho$ increases friction in the inflaton field equation and inflation at high energies proceeds at a higher rate than the corresponding rate in GR. Moreover, it was shown that, contrary to expectations, a large initial anisotropy introduces more damping into the scalar field equation of motion and results in more inflation [9].

At late(r) times a number of features of the cosmological dynamics can be deduced directly from Eqn (9), consistent with the qualitative analysis of perfect fluid RW and Bianchi type I and V cosmological models in the Randall-Sundrum brane-world scenario of [15]. Models with a positive curvature can recollapse. However, for $\mathcal{U}<0$ models can (re)collapse (even without a positive curvature) for any values of $\gamma$. Indeed, for $\mathcal{U}<0$ and positive curvature (as in the $k=1$ BRW models), there exist oscillating universes in which the physical variables 
oscillate periodically without reaching any spacelike singularity 15 (it was noted earlier that when there are bulk effects present a singularity can be avoided). When a positive cosmological constant is present, the de Sitter model is always the global attractor for $\mathcal{U} \geq 0$. For $\mathcal{U}<0$, models can (re)collapse (even without a positive curvature) so that in this case the de Sitter models is only a local attractor (and the cosmic no-hair theorem is consequently violated 16]).

Acknowledgements: This work was supported, in part, by NSERC of Canada.

* $\quad$ Electronic address: aac@mscs.dal.ca

[1] V. Rubakov and M. E. Shaposhnikov, Phys. Lett. B159, 22 (1985); J. Polchinsky, Phys. Rev. Lett. 75, 4724 (1995); P. Horava and E. Witten, Nucl. Phys. B460, 506 (1996); N. Arkani-Hamed, S. Dimopoulos and G. Dvali, Phys. Lett. B429, 263 (1998); I. Antoniades, N. ArkaniHamed, S. Dimopoulos and G. Dvali, Phys. Lett. B436, 257 (1998); A. Lukas, B. A. Ovrut and D. Waldram, Phys. Rev. D60, 086001 (1999).

[2] L. Randall and R. Sundrum, Phys. Rev. Lett. 83, 3370 (1999); ibid 83, 4690 (1999); N. Arkani-Hamed, S. Dimopoulos, G. Dvali and N. Kaloper, Phys. Rev. Lett. 84, 586 (2000); A. Chamblin and G. W. Gibbons, Phys. Rev. Lett. 84, 1090 (2000).

[3] T. Shiromizu, K. Maeda, and M. Sasaki, Phys. Rev. D 62, 024012 (2000); M. Sasaki, T. Shiromizu, and K. Maeda, Phys. Rev. D 62, 024008 (2000).

[4] R. Maartens, Phys. Rev. D 62, 084023 (2000).

[5] J. M. Cline, C. Grojean and G. Servant, Phys. Rev. Lett. 83, 4245 (1999); R. N. Mohapatra, A. Perez-Lorenzana and C. A. de S. Pires, Phys. Rev. D 62, 105030 (2000); C. Csaki, M. Graesser, C. Kolda, and J. Terning, Phys. Lett. B462, 34 (1999); D. Ida, J. High Energy Phys. 09, 014 (2000); N. Kaloper, Phys. Rev. D 60, 123506 (1999); P. Kanti, I.I. Kogan, K.A. Olive, and M. Pospelov, Phys. Lett. B468, 31 (1999); P. Kraus, JHEP 9912, 011 (1999); H. Stoica, S.-H. Henry Tye, and I. Wasserman, Phys. Lett. B482, 205 (2000); S. Mukohyama, T. Shiromizu, and K. Maeda, Phys. Rev. D 61, 024028 (2000); D. Langlois, R. Maartens, and D. Wands, Phys. Lett. B489, 259 (2000); C. M. Chen, T. Harko and M. K. Mak, Phys. Rev. D 64, 044013 (2001).

[6] P. Binétruy, C. Deffayet, and D. Langlois, Nucl. Phys. B565, 269 (2000); P. Binétruy, C. Deffayet, U. Ellwanger, and D. Langlois, Phys. Lett. B 477, 285 (2000).

[7] C.B. Collins and S.W. Hawking, Ap. J. 180, 317 (1973).

[8] L.G. Jensen and J. Stein-Schabes, Phys. Rev. D 34, 831 (1986); T. Rothman and G.F.R. Ellis, Phys. Lett. B180, 19 (1986).

[9] R. Maartens, V. Sahni and T. D. Saini, Phys. Rev. D 63, 063509 (2001).

[10] R. Maartens, D. Wands, B.A. Bassett, and I.P.C. Heard, Phys. Rev. D 62, 041301R (2000).
[11] E.M. Lifshitz and I.M. Khalatnikov, Adv. Phys. 12, 185 (1963); V A. Belinskii, I. M. Khalatnikov and E. M. Lifshitz, Adv. Phys. 19, 525 (1970); ibid Adv. Phys. 31, 639 (1982); V.A. Belinskii and I.M. Khalatnikov, Ž. Èksper. Teoret. Fiz. 63, 1121 (1972).

[12] J. Wainwright and G. F. R. Ellis (eds) Dynamical Systems in Cosmology (Cambridge: CUP 1997)

[13] A. D. Rendall, Class. Quantum Grav. 14, 2341 (1997); L. Andersson and A. D. Rendall, Comm. Math. Phys. 218, 479 (2001); H. Ringström, Class. Quantum Gravity $\mathbf{1 7}$ 713 (2000); ibid gr-qc/0006035.

[14] S.W. Goode and J. Wainwright, Class. Quantum Grav. 2, 99 (1985).

[15] A. Campos and C. F. Sopuerta, Phys. Rev. D 63, 104012 (2001); A. Campos and C. F. Sopuerta, Phys. Rev. D 64, 104011 (2001).

[16] M. G. Santos, F. Vernizzi, and P. G. Ferreira, Phys. Rev. D 64, 063506 (2001).

[17] A. A. Coley, Class. Quantum Grav. 19, L45 (2002).

[18] B. K. Berger, V. Moncrief, Phys. Rev. D 48, 4676 (1993); ibid, Phys. Rev. D 58, 064023 (1998); B. K. Berger and D. Garfinkle, Phys. Rev. D 57, 4767 (1998).

[19] M. Weaver, J. Isenberg and B. K. Berger, Phys. Rev. Letts. 80, 2984 (1998).

[20] J. D. Barrow, Nature 272, 211 (1978).

[21] R. Penrose, in General Relativity: An Einstein Centenary Survey, eds. S.W. Hawking and W. Israel (CUP 1979).

[22] S.W. Goode, A.A. Coley and J. Wainwright, Class. Quantum Grav. 9, 445 (1992). 